\title{
Diseño y Construcción de un Aerogenerador Tipo Savonius Multietapa
}

\author{
Design and Construction of a Savonius Multietapa Type Wind Turbine
}

\author{
Carlos Job Fiestas Urbina', Benigno Benito Lizárraga Zavaleta². José Vicente Nunja García², \\ Celso Quispe Ojeda ${ }^{3}$, Jorge Rojas Paz ${ }^{1}$
}

\begin{abstract}
RESUMEN
Objetivo. Construir y evaluar un sistema no convencional de generación de energía eléctrica aprovechando el potencial eólico disponible, y determinar los principios que intervienen en el diseño y el funcionamiento del sistema conformado por un aerogenerador de eje vertical tipo Savonius multietapa, el cual posee un rotor de dos etapas desfasadas con un ángulo de $90^{\circ}$ y un área de $0.704 \mathrm{~m}^{2}$. Material y Métodos. Para la construcción del prototipo se utilizó material reciclado de tableros de dibujo desechados por la Universidad, contenedores cilíndricos de aceite lubricante y piezas de acero maquinadas a medida. Los instrumentos de medida de los parámetros velocidad del viento y velocidad angular del rotor fueron respectivamente: Anemómetro digital portátil con unidades de $\mathrm{m} / \mathrm{s}$ con precisión de $\pm 3 \%$ del valor, y tacómetro digital láser portátil con unidades RPM con precisión de $\pm 0,003 \%, \pm 2$ dígitos. Resultados. La turbina fue evaluada en condiciones naturales con velocidades del viento en un rango de 1.2 a $5.0 \mathrm{~m} / \mathrm{s}$, a temperatura del aire $T=25^{\circ} \mathrm{C}$, y presión atmosférica $\mathrm{P}=$ 98171.4 Pa. Se obtuvieron para la turbina las correlaciones empíricas siguientes: Torque estático, vs. velocidad del viento $T s=0.3062 \mathrm{Vv}^{2}-0.2649 \mathrm{Vv}$ Velocidad angular, vs. velocidad del viento: $\omega=61.401-72.2331 \exp (-$ $0.4564 \mathrm{Vv}$ ) Conclusión. Con la ayuda de estas correlaciones, se logró obtener las curvas características del desempeño de la turbina: Coeficiente de potencia, vs. ratio típico de velocidad. $C_{p}=-0.9451 \lambda^{2}+1.4152 \lambda-$ 0.1328 Potencia eléctrica estimada, vs. velocidad del viento.
\end{abstract}

Palabras clave: Aerogenerador, tipo Savonius, construcción y evaluación.

\begin{abstract}
Objective: To construct and evaluate an unconventional system of electric power generation taking advantage of the available wind potential, and to determine the principles involved in the design and operation of the system consisting of a vertical axis wind turbine type Savonius multistage, which has a rotor Of two stages out of phase with an angle of $90^{\circ}$ and an area of $0.704 \mathrm{~m} 2$. Materials and methods: For the construction of the prototype was used recycled material of drawing boards discarded by the University, cylindrical containers of lubricating oil and pieces of steel machined to measure. The instruments of measurement of wind speed parameters and rotor angular velocity were respectively: Portable digital anemometer with $\mathrm{m} / \mathrm{s}$ units with $\pm 3 \%$ accuracy of value, and portable digital laser tachometer with RPM units with $\pm 0.003 \%, \pm 2$ digits. Results: The turbine was evaluated under natural conditions with wind speeds ranging from 1.2 to $5.0 \mathrm{~m} / \mathrm{s}$, at air temperature $\mathrm{T}=25^{\circ} \mathrm{C}$, and atmospheric pressure $\mathrm{P}=98171.4 \mathrm{~Pa}$. Empirical correlations were obtained for the turbine Following: $T s=0.3062$ $V_{v} v^{2}-0.2649 \mathrm{Vv}$ Static torque, vs. wind speed $\omega=61.401-72.2331 \exp (-0.4564 \mathrm{Vv})$ Conclusion: With the help of these correlations, it was possible to obtain the characteristic curves of the performance of the turbine: Power ratio, vs. typical rate of speed. $C_{p}=-0.9451 \lambda^{2}+1.4152 \lambda-0.1328$ Estimated electrical power, vs. wind speed.
\end{abstract}

Keyword: Wind turbine, Savonius type, construction and evaluation

\footnotetext{
Docente. Facultad de Ciencias. Universidad Nacional José Faustino Sánchez Carrión. Email: carfieur@yahoo.es

${ }^{2}$ Docente. Facultad de Ingeniería Química y Metalúrgica. Universidad Nacional José Faustino Sánchez Carrión.

${ }^{3}$ Docente. Facultad de Ingenieria Agraria, Industrias Alimentarias y Ambiental. Universidad Nacional José Faustino Sánchez Carrión.
} 


\section{INTRODUCCIÓN}

En el mundo actual se viene dando impulso a la investigación para el desarrollo de fuentes de energía no convencionales, a consecuencia de la escases cada vez mayor de hidrocarburos. Somos conocedores que en nuestra región, en zona costera y la serranía existe un potencial eólico para ser explotado a través de aerogeneradores de energía eléctrica.

Para continuar con el desarrollo de esta línea de investigación, en el presente proyecto proponemos construir y evaluar un aerogenerador de eje vertical tipo Savonius, y elaborar un procedimiento informatizado semiempírico para evaluar el desempeño del aerogenerador propuesto.

\section{Planteamiento y Formulación del problema}

Planteamiento del Problema:

Con la finalidad de contribuir con el desarrollo de tecnologías ensambladas en nuestros laboratorios, para aprovechar el potencial de energía eólica y convertirlo en energía eléctrica de mayor calidad medio ambiental, construiremos y evaluaremos un aerogenerador de eje vertical tipo Savonius, a nivel de prototipo para fines académicos y de investigación experimental, el cual se instalará dentro del campus universitario.

\section{Formulación del problema general:}

Son muy diversos los temas sobre los que hay que incidir y que intervendrán decididamente en el éxito de este estudio. La adaptación a las nuevas exigencias tiene que estar asentada en la formación profesional, la investigación y la innovación tecnológica; y en consecuencia, para definir el problema principal, se requiere responder las preguntas siguientes.

¿Será posible construir y evaluar, un sistema no convencional de generación de energía eléctrica aprovechando el potencial eólico disponible?

\section{Problemas Específicos:}

Para formular los problemas específicos, plantearemos las siguientes preguntas:

¿Qué principios intervienen en el funcionamiento de un sistema aerogenerador de energía eléctrica tipo Savonius multietapa?

¿Es posible diseñar, construir y evaluar un prototipo de aerogenerador de eje vertical tipo Savonius multietapa, para generar energía eléctrica a niveles de banco de laboratorio?

\section{Objetivos de la Investigación}

Objetivo Especifico 1. Determinar los principios que intervienen en el diseño y el funcionamiento de un aerogenerador de eje vertical tipo Savonius multietapa.

Objetivo Especifico 2. Construir un sistema no convencional de generación de energía eléctrica aprovechando el potencial eólico disponible.
Objetivo Especifico 3. Evaluar un prototipo de aerogenerador de eje vertical tipo Savonius, para generar energía eléctrica a niveles de banco de laboratorio.

\section{Antecedentes}

Se ha desarrollado previamente un procedimiento de simulación para evaluar un aerogenerador de eje horizontal de tipo toberas. El proyecto se denominó "DISEÑO Y EVALUACIÓN DE UN PROTOTIPO DE AEROGENERADOR USANDO TOBERAS", [Fiestas U., C.J. etAl. (2011)]

En el año 2013, en nuestro país, el 52\% de la producción de electricidad correspondió a la generación hidráulica, $46 \%$ vino de la energía térmica y solo el $2 \%$ proviene de recursos energéticos renovables, tales como: energía solar, energía eólica, energía proveniente de la biomasa, [Diario La República (13/10/2014), Pág.13].

El potencial eólico del país se estima en 22450 (MW). El geotérmico es de 3000 (MW). El potencial solar en la costa, sierra y selva del Perú va entre 4.5 y 6.5 (KW$H / \mathrm{m}^{2}$ ), [Diario La República (13/10/2014), Pág.13]

Se ha realizado un estudio para el diseño de aerobombas de eje vertical tipo Savonius en la Pontificia Universidad del Perú, [Miguel Hadzich (2008)].

[es.wikipedia.org/wiki/Energía_eólica (2014)] Existe en el mundo una gran cantidā de aerogeneradores operando, con una capacidad total de $318137 \mathrm{MW}$ a finales del 2013, de los que Europa cuenta con el 35 \% (2013). Estados Unidos y China, juntos, representan casi el $50 \%$ de la capacidad eólica global. Los primeros cinco países (EE.UU., China, Alemania, España e India) representaron $72,9 \%$ de la capacidad eólica mundial en 2009, ligeramente mayor que 72,4 $\%$ de 2008. La Asociación Mundial de Energía Eólica (World Wind Energy Association) anticipa que una capacidad de 200.000 MW será superada en el 2010.

Alemania, España, Estados Unidos, India y Dinamarca han realizado las mayores inversiones en generación de energía eólica. Dinamarca es, en términos relativos, la más destacada en cuanto a fabricación y utilización de turbinas eólicas, con el compromiso realizado en los años 1970 de llegar a obtener la mitad de la producción de energía del país mediante el viento. Actualmente genera más del $20 \%$ de su electricidad mediante aerogeneradores, mayor porcentaje que cualquier otro país, y es el quinto en producción total de energía eólica, a pesar de ser el país número 56 en cuanto a consumo eléctrico.

Definición de términos básicos [Gerónimo R., E. (2010)].

Aerogenerador eléctrico: Sistema tecnológico que permite convertir el potencial energético eólico, en primera etapa a energía mecánica rotacional y en segunda etapa en energía eléctrica. 
Energía: Magnitud física que permite evaluar la capacidad de un sistema para realizar trabajo sobre sí mismo y sobre el medio que lo rodea, en el Sistema Internacional de Unidades, se expresa en Julios (J).

Energía Convencional: La energía convencional es aquella que se obtiene de las siguientes fuentes: hidráulica, hidrocarburos, carbón.

Energía No Convencional: Es aquella que se obtiene de las fuentes consideradas no convencionales: nuclear, solar, eólica, geotérmica, mareomotriz, biomasa.

Energía Renovable: Es aquella energía cuya fuente potencial se renueva constantemente, con los ciclos de la naturaleza. Tal es el caso de las energías: hidráulica, solar, eólica, mareomotriz, geotérmica.

Energía no renovable: Es aquella energía cuya fuente potencial se agota con su explotación. Tal es el caso de las fuentes energéticas: hidrocarburos, carbón, nuclear.

Potencia: Es una magnitud física que permite evaluar la rapidez de realización de trabajo mecánico de un sistema. En el Sistema Internacional de Unidades se expresa en vatios (W).

\section{Estimación del recurso eólico.}

La cantidad de energía, ya sea mecánica o eléctrica, que puede generar una turbina eólica depende mucho de las características del viento vigente en el sitio de instalación.

De hecho, la producción puede variar de un sitio a otro, de manera que la rentabilidad de un proyecto depende directamente del recurso eólico local.

Figura $\mathbf{N}^{\circ}$ 01: A). (Izquierda), anemómetro portátil digital, con indicador en diferentes unidades $(\mathrm{m} / \mathrm{s}$, nudos, $\mathrm{km} / \mathrm{h}$, millas/h, ft/min)

- Rango: $0.4-30 \mathrm{~m} / \mathrm{s}$.

- Resolución: $0.1 \mathrm{~m} / \mathrm{s}$

- Precisión: $\pm 3 \%(<20 \mathrm{~m} / \mathrm{s})$ y $\pm 4 \%$ ( $>20 \mathrm{~m} / \mathrm{s})$.

B). (Derecha), tacómetro digital laser, con las siguientes especificaciones técnicas:

- Rango / Resolución:50 ... 99,99 / 0,01 rpm, 100 ... 9999,9 / 0,1 rpm, 10000 ... 99999 / 1 rpm

- Precisión: \pm 0,003 \% del valor ; \pm 2 dígitos

- Distancia de medición: 50 ... 250 mm

-Pantalla: LCD de 5 dígitos

- Desconexión automática: A los 30 segundos
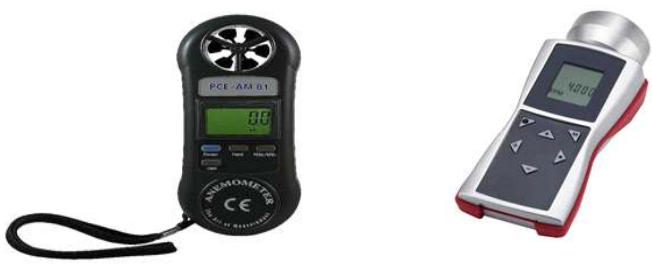

El método más exacto, aunque más costoso, para conocer el potencial de producción de energía del viento es la instalación de uno o más anemómetros, os cuales, periódicamente, generan datos de la velocidad y la dirección del viento en forma electrónica.

El anemómetro es un aparato meteorológico que se usa para la predicción del tiempo y, específicamente, para medir la velocidad del viento. No siempre es exacto a menos que sea un anemómetro digital.

Desde un punto de vista práctico, es el contenido energético del viento lo que interesa aprovechar. La energía cinética que transporta una masa de aire que se desplaza, viene determinada por la llamada ley de Betz o ley del cubo de la velocidad. Se puede expresar matemáticamente mediante la siguiente fórmula:

$$
\mathrm{P}=1 / 2 \rho \mathrm{AV}^{3}
$$

Donde:

$\mathrm{P}=$ energía cinética que atraviesa un área $\mathrm{A}$ transversal al flujo de aire, por unidad de tiempo, expresada en (W).

$A=$ área interceptada expresada en $\left(\mathrm{m}^{2}\right)$.

$\rho=$ densidad del aire expresada en $\left(\mathrm{kg} / \mathrm{m}^{3}\right)$.

$\mathrm{V}=$ velocidad del viento en $(\mathrm{m} / \mathrm{s})$.

Hay tres componentes del viento que determinan la potencia disponible de un sistema de conversión de energía eólica:

(1) Velocidad del viento, (2) densidad del aire atmosférico y (3) área interceptada por el aerogenerador.

Para pequeñas turbinas, la velocidad mínima requerida para el viento es aproximadamente de 3.5 $\mathrm{m} / \mathrm{s}$ y para turbinas grandes de $6 \mathrm{~m} / \mathrm{s}$ como mínimo.

\section{Conceptos básicos}

Los conceptos básicos han sido tomados de, Ali, M., H. (2013).

El desempeño de la turbina de eje vertical tipo Savonius puede ser explicada de acuerdo a las siguientes tres reglas básicas, aplicadas actualmente.

- La velocidad de los álabes es proporcional a la velocidad del viento.

- El máximo torque es proporcional al cuadrado de la velocidad del viento.

- La máxima potencia es proporcional al cubo de la velocidad del viento.

El desempeño de cualquier clase de turbina puede ser expresado en la forma de coeficiente de torque $\left(\mathrm{C}_{\mathrm{T}}\right)$ y el coeficiente de potencia $\left(C_{p}\right)$ en función del ratio típico de velocidad $(\lambda)$. 
El área de barrido: Para la turbina Savonius a partir de las dimensiones del rotor.

Figura $\mathbf{N}^{\circ}$ 02: Esquema del rotor de una etapa del aerogenerador de eje vertical Savonius

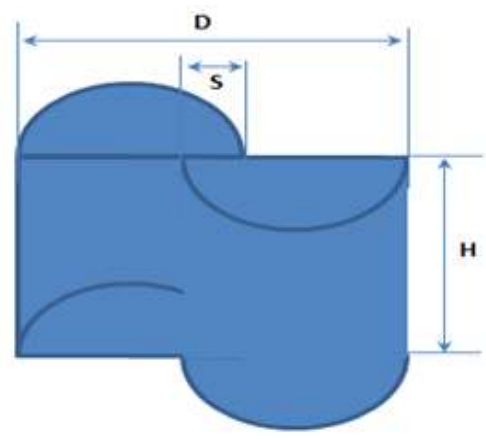

$$
A s=D \times H
$$

Donde:

As: área de barrido del rotor de la turbina Savonius, (m).

D: diámetro del rotor de la turbina Savonius, $(\mathrm{m})$.

$\mathrm{H}$ : altura del rotor de la turbina Savonius, $(\mathrm{m})$.

Ratio típico de velocidad: El ratio típico de velocidad, es la razón entre el producto de la velocidad angular del rotor por el radio del rotor y la velocidad del viento.

$$
\lambda=\frac{\omega\left(\frac{D}{2}\right)}{v v}
$$

Donde:

$\lambda$ : ratio típico de velocidad.

$\omega$ : velocidad angular del rotor, (rad/s).

D: diámetro del semicilindro del rotor Savonius, (m).

Vv: velocidad del viento, $(\mathrm{m} / \mathrm{s})$.

Coeficiente de torque: es el ratio entre el torque actual desarrollado por el rotor y el torque teórico disponible en el viento.

$$
\mathrm{C}_{\mathrm{ts}}=\frac{\mathrm{T}_{\mathrm{s}}}{\mathrm{T}_{\mathrm{w}}}=\frac{\mathrm{T}_{\mathrm{s}}}{0.25 \rho \mathrm{As} \mathrm{D} \mathrm{V}} \mathrm{V}_{v}^{2}
$$

También se usa el torque estático, el cual se define como el máximo torque en el rotor cuando este está impedido de rotar.

$$
\mathrm{C}_{\mathrm{T}}=\frac{\text { Torque del rotor }}{\text { Torque del viento }}=\frac{\mathrm{T}}{\mathrm{Tw}}=\frac{\mathrm{T}}{0.25 \rho \mathrm{As} \mathrm{d} \mathrm{V} v_{v}^{2}}
$$

Donde,

$\mathrm{C}_{\mathrm{T}}$ : coeficiente de torque.

$\mathrm{C}_{\mathrm{TS}}$ : coeficiente de torque estático.

$\mathrm{T}$ : torque del rotor, $(\mathrm{N} \mathrm{m})$.

Ts: torque estático, $(\mathrm{N} \mathrm{m})$.

Tw: torque disponible en el viento, ( $\mathrm{N} \mathrm{m})$.

$\rho:$ densidad del aire atmosférico, $\left(\mathrm{Kg} / \mathrm{m}^{3}\right)$.
As: área de barrido del rotor de la turbina Savonius, (m).

D: diámetro del semicilindro del rotor Savonius, (m).

Vv: velocidad del viento, $(\mathrm{m} / \mathrm{s})$.

Coeficiente de potencia: es el ratio de la máxima potencia obtenida del viento a la potencia disponible en el viento.

$$
\begin{gathered}
P w=T s \quad \omega \quad(6) \\
\mathrm{P} v_{v}=0.5 \rho H D V_{0}^{3} \\
C p=\frac{P w}{P v}=\frac{T s \omega}{0.5 \rho H D V_{0}^{3}}
\end{gathered}
$$

Donde:

$\mathrm{Cp}$ : coeficiente de potencia.

Pw: potencia obtenida del viento, (W).

Pv: potencia disponible en el viento, (W).

Ts: torque estático aplicado en el rotor, $(\mathrm{N} \mathrm{m})$.

$\omega$ : velocidad angular del rotor, (rad/s).

$\rho:$ densidad del aire atmosférico, $\left(\mathrm{Kg} / \mathrm{m}^{3}\right)$.

D: diámetro del rotor de la turbina Savonius, $(\mathrm{m})$.

$\mathrm{H}$ : altura del rotor de la turbina Savonius, $(\mathrm{m})$.

Vv: velocidad del viento, $(\mathrm{m} / \mathrm{s})$.

\section{Coeficiente de potencia máximo}

El máximo coeficiente de potencia se obtiene para valores del ratio típico de velocidad $(\lambda)$ próximos a la unidad.

Figura $\mathbf{N}^{\circ}$ 03: Gráfica del coeficiente de potencia (Cp) en función del ratio típico de velocidad $(\lambda)$, para turbinas Savonius de dos álabes.

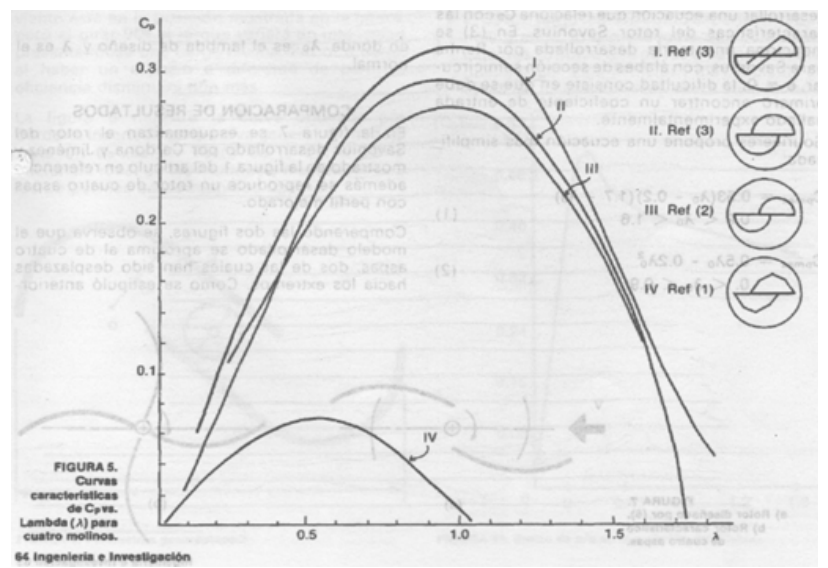

\section{MATERIALES Y MÉTODOS}

\section{Datos de evaluación}

\section{Datos iniciales}

Se emplearon los siguientes datos iniciales medidos en el lugar de la medición (pasaje frontal del laboratorio de Física I). 


\section{Tabla $N^{\circ} 01$}

Datos característicos de la turbina de eje vertical Savonius y de las condiciones meteorológicas durante la evaluación del aerogenerador.

\begin{tabular}{|c|c|c|}
\hline Temperatura del aire $(\mathrm{T})$ & $\begin{array}{r}25 \\
298.15\end{array}$ & $\begin{array}{l}\left({ }^{\circ} \mathrm{C}\right) \\
(\mathrm{K})\end{array}$ \\
\hline Presión atmosférica (Pat) & $\begin{array}{r}738 \\
98171.4168\end{array}$ & $\begin{array}{l}\text { (torr) } \\
(\mathrm{Pa})\end{array}$ \\
\hline Ancho del cilindro del rotor (D) & 1.05 & $(\mathrm{~m})$ \\
\hline Altura del rotor $(\mathrm{H})$ & 0.67 & $(\mathrm{~m})$ \\
\hline Número de etapas $(\mathrm{N})$ & 2 & \\
\hline $\begin{array}{l}\text { Longitud de traslape (s) } \\
\text { Area de la turbina (As) }\end{array}$ & 0.1 & $(\mathrm{~m})$ \\
\hline$A s=N * D * H$ & 1.407 & $\left(m^{2}\right)$ \\
\hline Peso molecular del aire (PM) & 0.02853 & $(\mathrm{Kg} / \mathrm{mol})$ \\
\hline $\begin{array}{l}\text { Constante universal de gases } \\
\text { ideales (R) }\end{array}$ & 8.3145 & $(\mathrm{~J} / \mathrm{mol} / \mathrm{K})$ \\
\hline $\begin{array}{l}\text { Rendimiento de conversión de } \\
\text { energía rotacional a eléctrica }\end{array}$ & 0.95 & (frac.) \\
\hline
\end{tabular}

Datos medidos para la evaluación del desempeño de la turbina de eje vertical tipo Savonius

\section{Densidad del aire}

La temperatura del aire medida en el momento de la evaluación $\mathrm{T}=25\left({ }^{\circ} \mathrm{C}\right)=298.15(\mathrm{~K})$.

La presión atmosférica en el momento de la evaluación $\mathrm{P}=736$ (torr) $=97805.57(\mathrm{~Pa})$.

La constante universal de los gases ideales es $\mathrm{R}=$ $8.3145(\mathrm{~J} / \mathrm{mol} / \mathrm{K})$.

El peso molecular del aire es $\mathrm{PM}=28.54(\mathrm{~g} / \mathrm{mol})$.

La densidad del aire se obtiene a partir de la siguiente expresión.

$$
\begin{gathered}
\rho=\left(\frac{P M}{R}\right) \frac{P}{T} \quad(9) \\
\rho=\frac{0.02854\left(\frac{K g}{m o l}\right) 97171.4168(P \boldsymbol{a})}{8.314\left(\frac{I}{m o l} K\right) 298.15(K)}=1.1187\left(\frac{K g}{m^{\prime}}\right)
\end{gathered}
$$

\section{Torque estático ejercido por el viento}

Empleando un dinamómetro, se mide el torque estático respecto al eje de giro.

\section{Tabla $N^{\circ} 02$}

Datos de torque estático en función de la velocidad

\begin{tabular}{|c|c|c|c|c|c|c|c|c|c|c|c|}
\hline $\mathbf{i}$ & $\begin{array}{c}\text { Vvi } \\
(\mathrm{m} / \mathrm{s})\end{array}$ & $\begin{array}{c}\mathbf{F i} \\
(\mathrm{N})\end{array}$ & $\begin{array}{c}\mathrm{Ti} \\
(\mathrm{Nm})\end{array}$ & i & $\begin{array}{c}\text { Vvi } \\
(\mathrm{m} / \mathrm{s})\end{array}$ & $\begin{array}{c}\mathrm{Fi} \\
(\mathrm{N})\end{array}$ & $\begin{array}{c}\mathrm{Ti} \\
(\mathrm{Nm})\end{array}$ & $\mathbf{i}$ & $\begin{array}{c}\text { Vvi } \\
(\mathrm{m} / \mathrm{s})\end{array}$ & $\begin{array}{c}\mathrm{Fi} \\
(\mathrm{N})\end{array}$ & $\begin{array}{c}\mathrm{Ti} \\
\text { (Nm) }\end{array}$ \\
\hline 1 & 1.6 & 1.1 & 0.578 & 31 & 2.5 & 1.7 & 0.893 & 61 & 2.4 & 1.7 & 0.893 \\
\hline 2 & 1.0 & 1.5 & 0.788 & 32 & 2.2 & 1.1 & 0.578 & 62 & 2.2 & 1.1 & 0.578 \\
\hline 3 & 1.7 & 1.3 & 0.683 & 33 & 2.0 & 1.1 & 0.578 & 63 & 3.0 & 2.5 & 1.313 \\
\hline 4 & 1.8 & 1.3 & 0.683 & 34 & 2.3 & 1.1 & 0.578 & 64 & 1.0 & 0.5 & 0.263 \\
\hline 5 & 1.7 & 1.1 & 0.578 & 35 & 2.4 & 1.3 & 0.683 & 65 & 2.0 & 1.1 & 0.578 \\
\hline 6 & 2.0 & 1.5 & 0.788 & 36 & 1.4 & 1.3 & 0.683 & 66 & 3.0 & 4.0 & 2.100 \\
\hline 7 & 1.3 & 0.7 & 0.368 & 37 & 2.1 & 1.1 & 0.578 & 67 & 3.6 & 5.0 & 2.625 \\
\hline 8 & 1.3 & 0.7 & 0.368 & 38 & 2.4 & 1.7 & 0.893 & 68 & 3.0 & 4.0 & 2.100 \\
\hline 9 & 1.5 & 0.8 & 0.420 & 39 & 1.4 & 1.3 & 0.683 & 69 & 4.0 & 5.0 & 2.625 \\
\hline 10 & 1.6 & 0.9 & 0.473 & 40 & 2.2 & 1.2 & 0.630 & 70 & 4.0 & 6.0 & 3.150 \\
\hline 11 & 1.5 & 1.0 & 0.525 & 41 & 2.4 & 1.7 & 0.893 & 71 & 3.0 & 4.0 & 2.100 \\
\hline 12 & 1.4 & 0.5 & 0.263 & 42 & 1.9 & 0.9 & 0.446 & 72 & 4.0 & 7.0 & 3.675 \\
\hline 13 & 0.9 & 0.5 & 0.263 & 43 & 1.4 & 0.7 & 0.368 & 73 & 5.0 & 9.5 & 4.988 \\
\hline 14 & 1.3 & 0.6 & 0.315 & 44 & 1.9 & 0.9 & 0.446 & 74 & 4.5 & 10.0 & 5.250 \\
\hline 15 & 1.9 & 1.1 & 0.578 & 45 & 1.6 & 0.8 & 0.394 & 75 & 2.5 & 3.0 & 1.575 \\
\hline 16 & 2.7 & 1.7 & 0.893 & 46 & 2.2 & 1.1 & 0.578 & 76 & 3.6 & 8.0 & 4.200 \\
\hline 17 & 2.0 & 2.1 & 1.103 & 47 & 1.5 & 0.6 & 0.315 & 77 & 3.6 & 7.8 & 4.095 \\
\hline 18 & 2.2 & 1.5 & 0.788 & 48 & 1.3 & 0.7 & 0.368 & 78 & 3.2 & 7.0 & 3.675 \\
\hline 19 & 2.3 & 1.6 & 0.840 & 49 & 2.3 & 1.1 & 0.578 & 79 & 4.0 & 10.0 & 5.250 \\
\hline 20 & 2.0 & 1.0 & 0.525 & 50 & 2.8 & 2.5 & 1.313 & 80 & 4.0 & 10.0 & 5.250 \\
\hline 21 & 2.4 & 1.6 & 0.840 & 51 & 2.4 & 1.6 & 0.840 & 81 & 2.5 & 4.0 & 2.100 \\
\hline 22 & 2.5 & 1.4 & 0.735 & 52 & 2.0 & 1.1 & 0.578 & 82 & 3.0 & 6.0 & 3.150 \\
\hline 23 & 2.2 & 1.2 & 0.630 & 53 & 1.6 & 0.8 & 0.420 & 83 & 3.0 & 5.5 & 2.888 \\
\hline 24 & 2.3 & 1.2 & 0.630 & 54 & 2.6 & 2.0 & 1.050 & 84 & 3.0 & 6.0 & 3.150 \\
\hline 25 & 2.8 & 1.5 & 0.788 & 55 & 2.5 & 1.8 & 0.919 & 85 & 4.0 & 9.5 & 4.988 \\
\hline 26 & 2.7 & 2.0 & 1.050 & 56 & 1.8 & 0.9 & 0.473 & 86 & 3.3 & 2.0 & 1.050 \\
\hline 27 & 3.5 & 2.5 & 1.313 & 57 & 1.1 & 0.6 & 0.289 & 87 & 3.0 & 7.0 & 3.675 \\
\hline 28 & 2.7 & 1.7 & 0.893 & 58 & 2.4 & 1.7 & 0.893 & & & & \\
\hline 29 & 2.3 & 1.1 & 0.578 & 59 & 2.0 & 1.0 & 0.525 & & & & \\
\hline 30 & 2.1 & 1.1 & 0.578 & 60 & 2.3 & 1.8 & 0.945 & & & & \\
\hline
\end{tabular}
del viento.
El dinamómetro se ubica en el borde externo de la turbina cilíndrica perpendicularmente al plano transversal al flujo del aire incidente. Se mide la fuerza necesaria para impedir la rotación de la turbina que corresponde a una determinada velocidad de viento. Esta fuerza luego se multiplica por la distancia desde el eje de giro al punto donde está ubicado el manómetro $(\mathrm{D} / 2)$.

Figura N04: Gráfica de los datos de torque estático $\checkmark s$ velocidad del viento, y su correlación empírica.

Torque y Velocidad del Viento

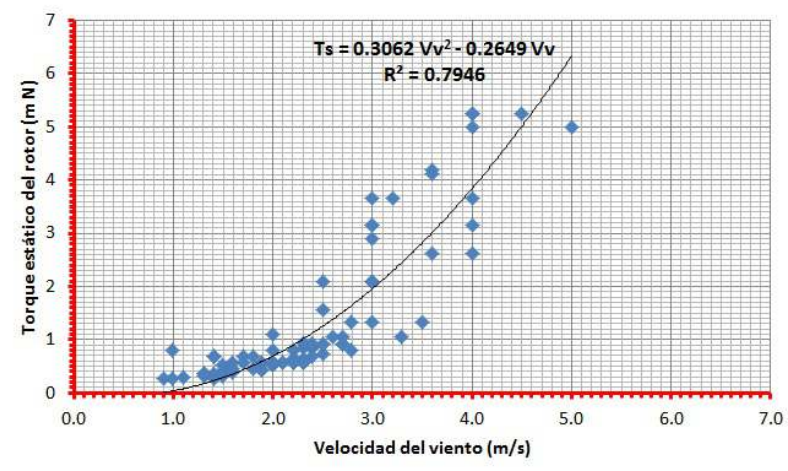

El resultado del ajuste de datos, es la ecuación empírica

$$
T s=0.3062 V v^{2}-0.2649 V v
$$

\section{Velocidad angular del rotor causada por el viento}

Empleando el tacómetro digital, se midió la velocidad angular del rotor del aerogenerador correspondiente a cada velocidad del viento, la velocidad del viento se midió usando el anemómetro digital. Se aprovechó las 
ráfagas de viento que ocurren a diferentes horas, frente al laboratorio de Física I de la UNJFSC.

\section{Tabla $N^{\circ} 03$}

Datos de velocidad angular del rotor en función de la velocidad del viento

\begin{tabular}{ccccc}
\hline $\mathbf{i}$ & $\begin{array}{c}\mathbf{V} \mathbf{v i} \\
(\mathbf{m} / \mathbf{s})\end{array}$ & $\begin{array}{c}\boldsymbol{\omega} \mathbf{i} \\
(\mathbf{r p m})\end{array}$ & $\begin{array}{c}\langle\boldsymbol{\omega}>\mathbf{i} \\
(\mathbf{r p m})\end{array}$ & $\begin{array}{c}{\left[(\boldsymbol{\omega} \mathbf{i}-\langle\boldsymbol{\omega} \mathbf{i}) /<\omega>\mathbf{i}]^{2}\right.} \\
(\mathbf{r p m})^{2}\end{array}$ \\
\hline 1 & 4.00 & 49.00 & 49.76 & 0.000234673 \\
2 & 1.00 & 18.00 & 15.64 & 0.022846357 \\
3 & 1.20 & 19.30 & 19.63 & 0.000280692 \\
4 & 1.90 & 30.10 & 31.05 & 0.000940383 \\
5 & 2.00 & 29.00 & 32.41 & 0.01104799 \\
6 & 2.10 & 33.00 & 33.70 & 0.000431155 \\
7 & 2.20 & 34.00 & 34.94 & 0.000717199 \\
8 & 2.20 & 32.00 & 34.94 & 0.007060836 \\
9 & 2.50 & 38.00 & 38.32 & $7.06224 \mathrm{E}-05$ \\
10 & 2.50 & 37.00 & 38.32 & 0.001190136 \\
11 & 2.80 & 43.00 & 41.28 & 0.00174627 \\
12 & 2.90 & 45.00 & 42.17 & 0.004493288 \\
13 & 3.00 & 43.00 & 43.03 & $5.14915 \mathrm{E} .07$ \\
14 & 3.00 & 44.00 & 43.03 & 0.00050722 \\
15 & 3.00 & 45.00 & 43.03 & 0.002094039 \\
16 & 3.00 & 46.00 & 43.03 & 0.004760973 \\
17 & 3.30 & 47.00 & 45.38 & 0.001271983 \\
18 & 3.30 & 45.80 & 45.38 & $8.50521 \mathrm{E}-05$ \\
19 & 3.50 & 46.00 & 46.78 & 0.000277285 \\
20 & 3.50 & 48.00 & 46.78 & 0.000681334 \\
21 & 3.60 & 48.00 & 47.43 & 0.000143762 \\
22 & 3.60 & 47.00 & 47.43 & $8.26836 \mathrm{E}-05$ \\
23 & 4.00 & 49.50 & 49.76 & $2.77865 \mathrm{E}-05$ \\
24 & 4.10 & 49.00 & 50.28 & 0.000649613 \\
25 & 4.40 & 52.00 & 51.70 & $3.26924 \mathrm{E}-05$ \\
26 & 4.60 & 51.00 & 52.55 & 0.000870286 \\
27 & 4.70 & 52.00 & 52.95 & 0.000318659 \\
\hline $\boldsymbol{\Sigma}$ & & & & $\mathbf{0 . 0 6 2 8 6 3 4 8 7}$ \\
\hline
\end{tabular}

la ecuación de la forma, $\mathrm{Y}(\mathrm{x})=\mathrm{A}+\mathrm{B} \exp (-\mathrm{Cx})$.

Figura Nº5: Gráfica de los datos de velocidad angular del rotor del aerogenerador Vs velocidad del viento, y su correlación empírica.

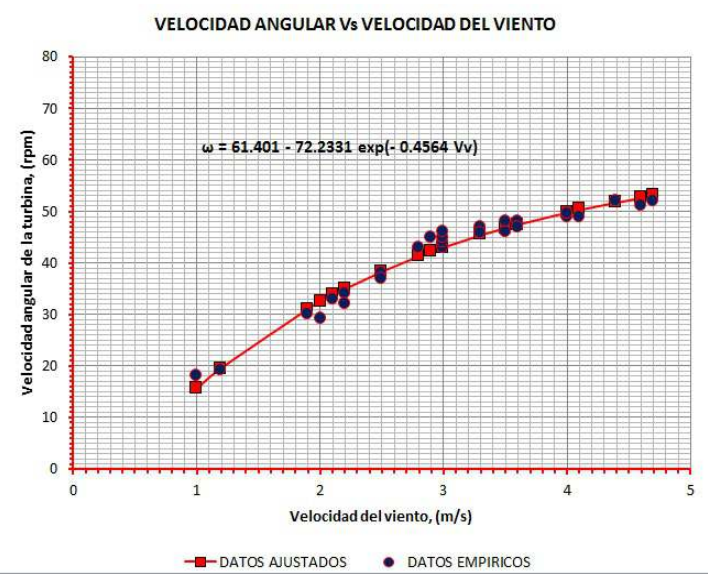

El ajuste de los datos a la función escogida se realizó utilizando una aplicación en EXCEL que emplea un método de Newton Raphson de aproximaciones sucesivas para ajustar funciones trascendentes no lineales.

El resultado del ajuste de datos es la ecuación empírica.

$$
\omega=61.401-72.2331 \exp (-0.4564 V v)
$$

\section{DISCUSION}

Haciendo uso de la correlaciones empíricas (10) y (11), podemos obtener varios parámetros característicos de la turbina del aerogenerador descritos en el marco teórico. Estos parámetros los podemos obtener en función de la velocidad del viento, como se muestra en la Tabla $\mathrm{N}^{\circ} 04$.

\section{Tabla $N^{\circ} 04$}

Parámetros de desempeño mecánico-eléctrico, estimados para la turbina del aerogenerador tipo Savonius

\begin{tabular}{|c|c|c|c|c|c|c|c|c|c|c|}
\hline \multicolumn{2}{|c|}{$\begin{array}{l}\text { I VELOCIDAD } \\
\text { DELARARS }\end{array}$} & $\begin{array}{l}\text { VELOOCIDAD } \\
\text { ANGULAR }\end{array}$ & $\begin{array}{c}\text { RATIO } \\
\text { TIPIICO DE } \\
\text { VELOCIDAD }\end{array}$ & $\begin{array}{l}\text { TORQUEE } \\
\text { ESTATICO }\end{array}$ & $\begin{array}{c}\text { TRRQUEE } \\
\text { TEORICO } \\
\text { DISPONBLE } \\
\text { EN EL VIENTO }\end{array}$ & $\begin{array}{l}\text { COEFICIENTE } \\
\text { DE TORQUE } \\
\text { ESTATICO }\end{array}$ & $\begin{array}{l}\text { POTENCIA } \\
\text { TEOOICA } \\
\text { DEL AIRE }\end{array}$ & $\begin{array}{l}\text { POTENCIA } \\
\text { EXTRADA } \\
\text { DEL VIENTO }\end{array}$ & $\begin{array}{l}\text { COEFFICENTE } \\
\text { DE POTENCIA }\end{array}$ & $\begin{array}{l}\text { POTENCIA } \\
\text { ELEECTIICA } \\
\text { GENERADA }\end{array}$ \\
\hline & $(\mathrm{m} / \mathrm{s})$ & $(\mathrm{rad} / \mathrm{s})$ & $($ s.u. $)$ & $(\mathrm{N} \mathrm{m})$ & $(\mathrm{N} \mathrm{m})$ & $(\mathrm{N} \mathrm{m})$ & (W) & (W) & (s.u.) & (W) \\
\hline & $\begin{array}{l}2.00 \\
250\end{array}$ & $\begin{array}{l}3.39 \\
401\end{array}$ & $\begin{array}{l}0.89 \\
0.84\end{array}$ & & & 0.42 & 6.36 & 2.35 & 0.37 & 2.24 \\
\hline & & & & & & & & & & $\begin{array}{l}4.62 \\
819\end{array}$ \\
\hline & 3.50 & $\begin{array}{l}4.91 \\
4.90\end{array}$ & 0.7 & $\begin{array}{l}1.791 \\
2.79\end{array}$ & & 0.55 & 34. & $\begin{array}{l}8.01 \\
13.68\end{array}$ & & 8.18 \\
\hline & 4.00 & 5.21 & & 3.86 & & & & & & $\begin{array}{l}13.00 \\
19.10\end{array}$ \\
\hline & 4.50 & & & & & & & 27. & & 26.48 \\
\hline & 5.00 & & & 6.53 & & & & & 0.37 & 35.12 \\
\hline & & 5.8 & & & & & & 47. & 0.36 & 45.00 \\
\hline & & & & & & & & & & 56.09 \\
\hline & 6.50 & 6.04 & 0.4 & $\begin{array}{l}11.92 \\
1407\end{array}$ & & & & & 0.33 & 68.37 \\
\hline & 7.50 & & & & & & & & & \\
\hline & 8.00 & & & & & & & & & \\
\hline & 8.50 & 6.27 & 0.39 & $\begin{array}{r}21.64 \\
\end{array}$ & 30.15 & 0.72 & 488.13 & 135.78 & 0.28 & $\begin{array}{l}112.110 \\
128.99\end{array}$ \\
\hline
\end{tabular}

De los datos de la tabla se pueden confeccionar dos gráficas importantes mostradas en la Figura $\mathrm{N}^{\circ} 06$, y Figura $\mathrm{N}^{\circ} 07$, que nos permiten evaluar el desempeño de la turbina para diferentes velocidades del viento.

Figura N 06: Gráfica que muestra el coeficiente potencia en función de ratio típico de velocidad, y su correspondiente ecuación empírica.

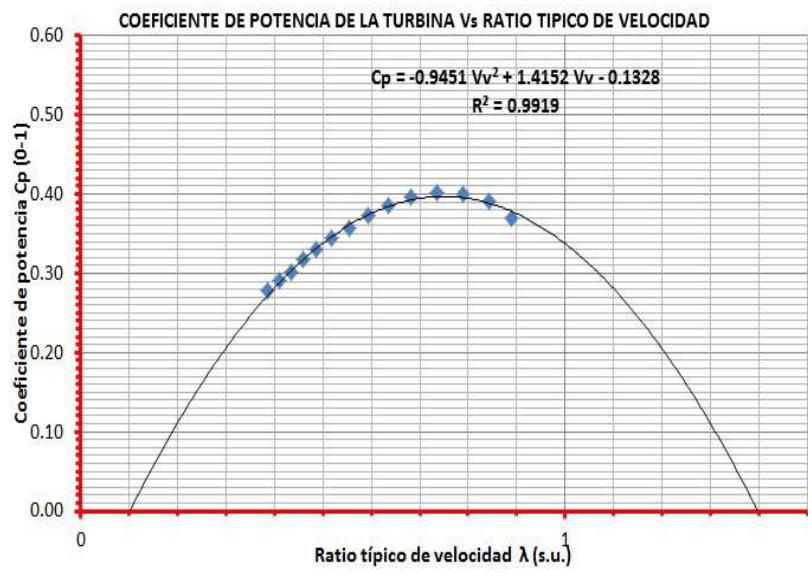

Se obtuvo la ecuación empírica que relaciona el coeficiente de potencia con el ratio típico de velocidad, de la turbina del aerogenerador tipo Savonius.

$$
C p=-0.9451 V v^{2}+1.4152 V v-0.1328
$$

También se obtuvo la ecuación empírica que relaciona la potencia eléctrica que se podría extraer de la turbina del generador en función de la velocidad del viento.

POTel $=-0.0003 V v^{6}+0.0095 V v^{5}-0.1383 V v^{4}+1.0147 V v^{3}-1.3972 V v^{2}$ $+0.8149 \mathrm{Vv}$

(13) 
Figura $\mathbf{N}^{\circ}$ 07: Gráfica que muestra la potencia eléctrica en función de la velocidad del viento y su correspondiente ecuación empírica.

\section{POTENCIA ELECTRICA VS VELOCIDAD VIENTO}

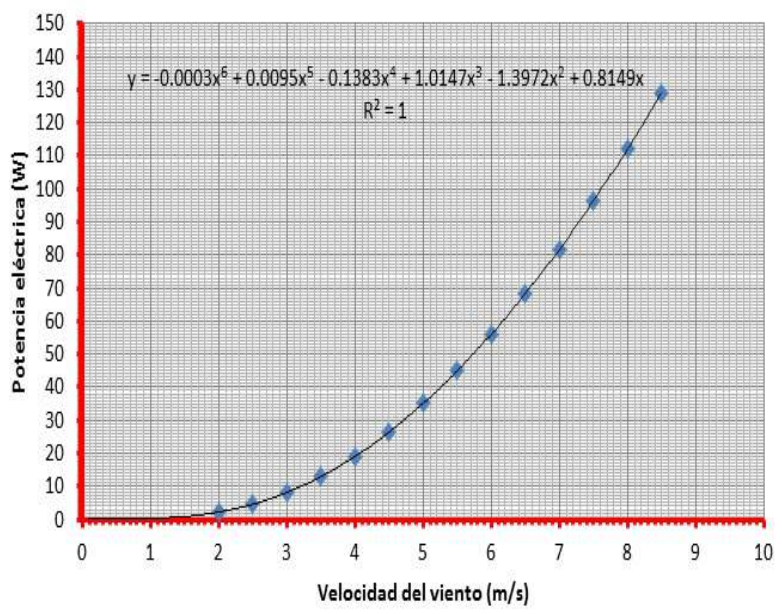

\section{CONCLUSIONES}

- Se ha diseñado y construido, un aerogenerador de eje vertical tipo Savonius, utilizando material reciclado.

- Se ha evaluado el desempeño de la turbina (rotor) del aerogenerador, para diferentes velocidades del viento, en el rango 1 - $5(\mathrm{~m} / \mathrm{s})$, temperatura del aire T $=25\left({ }^{\circ} \mathrm{C}\right)$, presión atmosférica $\mathrm{P}=98171.4168(\mathrm{PA})$, obteniéndose ecuaciones empíricas que permiten evaluar el desempeño de la turbina para diferentes velocidades del viento.

- Cp Vs Vv:

$$
C p=-0.9451 V v^{2}+1.4152 v v-0.1328
$$

- POTel Vs Vv:

$$
\begin{aligned}
\text { POTel }= & -0.0003 V v^{6}+0.0095 V v^{5}-0.1383 V v^{4}+1.0147 V v^{3} \\
& -1.3972 V v^{2}+0.8149 V v
\end{aligned}
$$

\section{AGRADECIMIENTO}

Agradecemos la contribución de la Tesista Suzana Jacqueline Ramírez Pretel por su donación de un anemómetro digital, contribución que fue de mucha utilidad para el desarrollo de la investigación.

Asimismo, agradecemos al Vicerrectorado de Investigación de la Universidad Nacional José Faustino Sánchez Carrión, por la publicación del presente artículo en la Revista Big Bang.

\section{REFERENCIAS BIBLIOGRÁFICAS Y BIBLIOGRAFÍA DE APOYO}

\section{A) REFERENCIAS BIBLIOGRÁFICAS}

Fiestas, C., J., Lizárraga, B., B., Valencia, J., C. (2011). Diseño y evaluación de un prototipo de aerogenerador usando toberas. Proyecto de investigación FEDU-2010. Universidad Nacional José Faustino Sánchez Carrión.

Gerónimo, Redondo, E. (2010). Fundamentos de la generación eléctrica mediante energía eólica. Universidad Veracruzana, Facultad de Ingeniería Mecánica Eléctrica. Veracruz, México.

Hadzich, M. (2001). Curso de energía eólica: Diseño de aerobombas Savonius. Universidad Católica del Perú. Lima.

Internet Libre (consultado 20 de Octubre del 2014), disponible:

http://es.wikipedia.org/wiki/energi\%C3\%ADa_e\%C3 \%B31lica.

Diario La República (13/OCT/2014). Pág. 13. Lima Perú.

Ali, M., H. (2013). Experimental comparison study for Savonius wind turbine of two \& three blades at low wind speed. International Journal of Modern Engineering Research. ISSN 2249-6645. Vol. 3, Issue. 5, Sep-Oct 2013, pp-2978-2986.

Rodríguez Devis, Julio, M. (1985). Coeficientes de potencia en molinos Savonius. Ingeniería e Investigación. Volumen $3 \mathrm{~N}^{\circ} 4$, trimestre 4-1985, pp-62-67.

\section{B. BIBLIOGRAFÍA DEAPOYO}

Shames, V. (2010). La mecánica de los fluidos. Edit. McGraw-Hill.9² Edición. México D. F.

Streeter, I., M. (2010). Mecánica de fluidos. Edit. McGraw-Hill. $3^{\circ}$ Edición. México, D. F.

Giles R. (2014). Mecánica de fluidos e hidráulica. Edit. Schaum-McGraw-Hill.

\section{ANEXOS FOTOS DEL DESARROLLO DE LA TURBINASAVONIUS}

Figura $\mathbf{N}^{\circ}$ 07: Presentación de la turbina Savonius de dos etapas (RHINO-1)

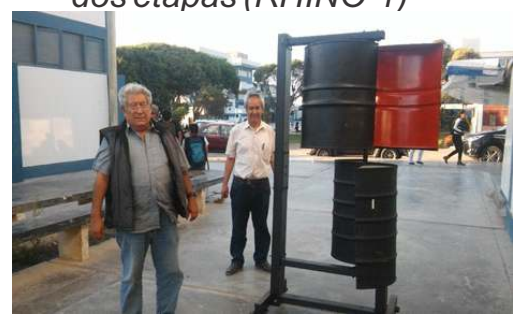

Figura $\mathbf{N}^{\circ}$ 08: Realizando las mediciones en el campus universitario.

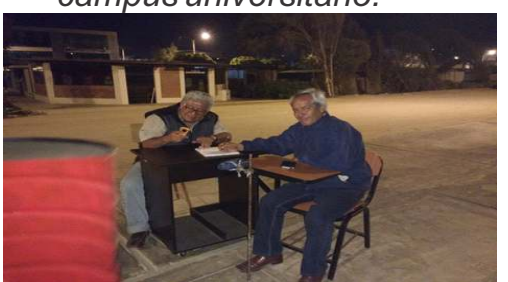

Octubre - Diciembre 2016 